\title{
LA CONVERGENCIA TECNOLÓGICA EN LAS AULAS VIRTUALES DE LA FACULTAD DE ESTUDIOS A DISTANCIA DE LA UNIVERSIDAD MILITAR NUEVA GRANADA (UMNG) ${ }^{1}$
}

\author{
PAULA COLORADO ORDOÑZZ2; JOSÉ GUILLERMO COGOLLO RINCÓN³ \\ UNIVERSIDAD MILITAR NUEVA GRANADA (UMNG)
}

Recibido, agosto 29 de 2014

Concepto evaluación, septiembre 29 de 2014

Aceptado, octubre 07 de 2014

\author{
Referencia: Colorado Ordóñez, P.; Cogollo \\ Rincón, J.G., (2014). "La convergencia \\ tecnológica en las aulas virtuales de la facultad \\ de estudios a distancia de la Universidad \\ Militar Nueva Granada". Revista Academia y \\ Virtualidad, 7, (2), 112-120
}

\section{Resumen}

El presente trabajo analiza si la comunidad académica de la Facultad de Estudios a Distancia está preparada para una convergencia tecnológica en cuanto a los contenidos virtuales de las asignaturas. El objeto del artículo es estudiar la viabilidad de ofrecer todos los contenidos virtuales a través de dispositivos móviles y, de esta manera, tener mayor cobertura para los procesos de formación.

Para este estudio se encuestaron docentes y estudiantes del aula virtual de la Facultad de Estudios a Distancia, se efectuó análisis cuantitativo de la información recolectada con el uso de herramientas tecnológicas y se tomaron los elementos motivo de estudio, para hacer el análisis correspondiente. Los estudiantes y docentes mostraron que tienen habilidades en el uso de dispositivos electrónicos, móviles y entornos virtuales, las cuales se pueden reflejar en el entorno académico para conseguir una educación de mayor calidad.

Palabras clave: convergencia, dispositivos móviles, aula virtual, contenidos virtuales.

1. Artículo de investigación científica y tecnológica, resultado del proyecto financiado por el Fondo de Investigaciones de la UMNG, DIS-1244

2. Docente, Universidad Militar Nueva Granada; Especialista, Alta Gerencia, Universidad Militar Nueva Granada. Correo: paula.colorado@unimilitar. edu.co

3. Docente, Universidad Militar Nueva granada; Maestría, Educación, Universidad Militar Nueva Granada. Correo: jose.cogollo@unimilitar.edu.co 
La convergencia tecnológica en las aulas virtuales de la facultad de estudios a distancia de la universidad militar nueva granada (UMNG)

\title{
THE TECHNOLOGICAL CONVERGENCE IN THE VIRTUAL CLASSROOMS AT THE FACULTY OF DISTANCE EDUCATION AT UNIVERSIDAD MILITAR NUEVA GRANADA (UMNG)
}

\begin{abstract}
This paper work analyzes whether the academic community of the Online Education Faculty if is ready to do technological convergence on the virtual course content. The purpose of the article is to study the feasibility of offering all virtual contents through mobile devices, and this way to have more coverage for formation processes.

This study was based in teachers and students surveyed through the virtual platform of Online Education Faculty; a qualitative analysis of the information collected through the use of technological tools and the object of study items were taken to the respective analysis was made. Teachers and Students showed skills in the use of electronic items, as mobile devices and virtual environments that can be reflected in the academic environment to achieve a higher quality education.
\end{abstract}

Keywords: convergence, mobile devices, virtual classroom, virtual content.

\section{A CONVERGÊNCIA TECNOLÓGICA NAS AULAS VIRTUAIS DA FACULDADE DE ESTUDOS A DISTÂNCIA DA UNIVERSIDADE MILITAR NUEVA GRANADA (UMNG)}

\section{Resumo}

O presente trabalho analisa se a comunidade acadêmica da Faculdade de Estudos a Distância está preparada para uma convergência tecnológica em quanto aos conteúdos virtuais das matérias. $\mathrm{O}$ objeto do artigo é estudar a viabilidade de oferecer todos os conteúdos virtuais através de dispositivos móveis e, deste jeito, ter maior cobertura para os processos de formação.

Para este estudo se entrevistaram docentes e estudantes da aula virtual da Faculdade de Estudos a Distância, se efetuou análise quantitativa da informação recolhida com uso de ferramentas tecnológicas e se tomaram os elementos motivo do estudo, parar fazer a análise correspondente. Os estudantes e docentes mostraram que têm habilidades no uso de dispositivos eletrônicos, móveis e entornos virtuais, as quais podem se refletir no entorno acadêmico para conseguir uma educação de maior qualidade.

Palavras-chave: convergência, dispositivos móveis, aula virtual, conteúdos virtuais. 


\section{Introducción}

En la actualidad el ritmo acelerado de la vida, las distancias, las obligaciones personales, sobre todo para las mujeres, no permiten que los individuos estudien y trabajen de manera presencial a la vez, por eso ahora tiene más acogida e importancia la educación a distancia; esta modalidad favorece a los estudiantes que son miembros de las fuerzas militares que por razones del servicio se encuentran en áreas apartadas en la geografía colombiana y que pueden acceder a esta educación gracias a las ventajas que ofrece las tecnologías de la información y las comunicaciones (TIC).

La importancia de ofertar programas a distancia a través de las nuevas tecnologías, radica en la conveniencia o no de llevar a cabo una innovación en los procesos de enseñanza-aprendizaje, o de continuar utilizando las bases teóricas de la educación presencial o a distancia soportadas en una enseñanza virtual. Con un desarrollo tecnológico tan apresurado como el que actualmente se está produciendo se pone aún más en evidencia el retraso que suponen algunas de las características de la educación formal y la necesidad de impartir una enseñanza de calidad a través del uso de las nuevas tecnologías, las cuales están fomentando la creación de nuevos modelos de formación uniendo teorías de aprendizaje, adoptadas de la educación a distancia, y creando progresivamente las nuevas bases teóricas para la fundamentación de una enseñanza multimedia interactiva y móvil.

Evidentemente, han cambiado los medios, los soportes en los que se almacenan los contenidos, la relación entre docentes y estudiantes y, en consecuencia, debe cambiar la enseñanza. (Ortega, 2004). De otro lado, las diferencias entre la educación a distancia y la educación a distancia a través de las nuevas tecnologías, se puede apreciar en la tabla 1. Aquí resulta importante precisar de acuerdo con la información anterior y del contexto Blended Learning de la UMNG, el cual se pone a consideración en el estudio, que la forma de interactuar del estudiante y del docente tutor para su aprendizaje autónomo, está dada por la mediación tecnológica basada en contenidos y recursos pedagógicos y didácticos especializados para favorecer este entorno; al respecto, las aulas virtuales (AVAS) con sus recursos y contenidos objetos de aprendizaje OVAS permiten componentes que a niveles de información digital se ofrecen de manera visible y auditiva para inducir al estudiante, como un atractivo más en brindar la información necesaria para el aprendizaje de sus competencias en temas específicos; sin embargo, no se debe dejar a un lado el tema de la ubicuidad que permite la disponibilidad de la información desde cualquier dispositivo, lugar y momento para ser consultado.

Asimismo, las mejoras introducidas en los entornos de formación a través de internet por el uso de las TIC, se han materializado en la aparición de plataformas de teleformación (aulas virtuales), sistemas hipermedia adaptativos y el aprendizaje colaborativo soportado por computador; no obstante, a pesar de ello, no se ha explotado al máximo su aplicación como medios didácticos en sistemas de aprendizaje más efectivos (Ramírez, 2011).

\begin{tabular}{|c|c|}
\hline Enseñanza a distancia & Enseñanza a distancia a través de las tecnología s \\
\hline Centrada en los contenidos & Centrada en el proceso \\
\hline Qué estudiar & Cómo estudiarlo \\
\hline Estudio individual y colaborativo & Estudio autónomo y colaborativo \\
\hline Enseñanza centrada en el profesor & Enseñanza centrada en el alumno \\
\hline Profesor como experto e instructor & Profesor como mediador, guía y orientador del aprendizaj e \\
\hline Alumno como receptor de información & Alumno como receptor y emisor de la información \\
\hline Limitaciones espacio-temporales & Superación de barreras espacio -temporales \\
\hline Comunicación bidireccional diferida & $\begin{array}{l}\text { Comunicación educativa tridireccional diferida y en tiempo } \\
\text { real }\end{array}$ \\
\hline $\begin{array}{l}\text { Profesor-tutor } \\
\text { Profesor-alumno } \\
\text { Tutor-alumno } \\
\text { Alumno-alumno }\end{array}$ & Profesor-tutor telemátic o-alumno \\
\hline $\begin{array}{l}\text { Tutorías semanales: apoyo tutorial } \\
\text { Libros de texto } \\
\text { Medios tecnológicos como apoyo al estudio } \\
\text { Emisiones de radio }\end{array}$ & $\begin{array}{l}\text { Tutorías semanales: apoyo tutorial } \\
\text { Aula virtual todos los días. Apoyo telemático } \\
\text { Libros de texto } \\
\text { Medios t ecnológicos como i nstrumentos facilitadores d el } \\
\text { aprendizaje } \\
\text { Emisiones de radio (descargadas a través de internet ) }\end{array}$ \\
\hline Videos & $\begin{array}{l}\text { Videoconferencias (descargadas a través de internet) } \\
\text { Internet } \\
\text { Cursos virtuales } \\
\text { Libro electrónico } \\
\text { CD-DVD-ROM }\end{array}$ \\
\hline Adquisición de conocimientos & $\begin{array}{l}\text { Adquisición de conocimientos, actitudes y habilidades de } \\
\text { interacción social m ediante las herramientas d e } \\
\text { comunicación }\end{array}$ \\
\hline Genera conocimientos & $\begin{array}{l}\text { Genera conocimientos, pensamiento creativo y critico ante } \\
\text { los medios tecnológicos }\end{array}$ \\
\hline Aprendizaje por objetivos & Aprendizaje por tareas \\
\hline $\begin{array}{l}\text { Se evalúa a través de exámenes escrito } \mathrm{s} \\
\text { Evaluación individual }\end{array}$ & $\begin{array}{l}\text { Se e valúa a través de m edios tecnológicos: } \\
\text { autoevaluaciones, trabajos, etc. } \\
\text { Evaluación individual y también grupal }\end{array}$ \\
\hline $\begin{array}{l}\text { Educación académica } \\
\text { Alfabetización }\end{array}$ & $\begin{array}{l}\text { Educación académica y tecnológica } \\
\text { Alfabetización tecnológica } \\
\text { Educación permanente }\end{array}$ \\
\hline
\end{tabular}

Tabla 1. Diferencias entre la educación a distancia y la educación a distancia a través de las nuevas tecnologías

Fuente: Ortega, 2004. 
La convergencia tecnológica en las aulas virtuales de la facultad de estudios a distancia de la universidad militar nueva granada (UMNG)

Las asignaturas en línea y la enseñanza móvil hacen que el alumno pase de un modelo pasivo (el de la clase tradicional) a uno proactivo, en el que puedan repasar, transformar y asimilar de forma diferente los contenidos (con recursos como audio, video, animación y gráficos), comenta Guereca Lozano, rector de la Universidad Interamericana para el Desarrollo (unid). En un modelo convencional, el alumno debe repasar sus notas de clase, con el digital refresca y actualiza sus conocimientos viendo un video (Vargas, 2010).

García Aretio (2001) define la educación a distancia como:

\section{... un sistema tecnológico de comunicación bidireccional (multidireccional), que puede ser masivo, basado en la acción sistemática y conjunta de recursos didácticos y el apoyo de una organización y tutoría, que, separados físicamente de los estudiantes, propician en éstos un aprendizaje independiente (cooperativo) (p. 39).}

Tratándose de los avances de internet aplicados a la educación se han visto grandes transformaciones, dada la tecnología que ofrece múltiples alternativas para el desarrollo e implementación de recursos pedagógicos aplicados tanto a modalidad presencial como a distancia, siendo esta última la que ha tenido mayores beneficios, ambientes que han recibido sus nombres según los avances tecnológicos, siendo los más comunes: e-Learning, para referirse al aprendizaje apoyado por recursos y herramientas electrónicas digitales, Blended Learning (b-Learning) es un ambiente combinado o mixto, es decir semipresencial y M_Learning es el e-Learning que se apoya de dispositivos móviles y transmisión de wireless; o simplemente, es cuando el aprendizaje toma lugar con dispositivos móviles.

La penetración de los recursos tecnológicos que apoyan los procesos de aprendizaje móvil han tenido influencias paralelas desde diversas vertientes; por un lado se encuentra el desarrollo de los servicios de comunicación, conectividad, facilidad de acceso a la información por las redes inalámbricas, cantidad de dispositivos móviles que aparecen en el mercado y hasta las prácticas de la sociedad actual donde la movilidad, los tiempos de traslados y esperas han provocado la opción de recursos para la productividad. (Ramírez, 2009).

Es como algunas instituciones universitarias que ofrecen educación presencial han iniciado el proceso de incursionar en la modalidad a distancia dado que presenta más acogida dentro del público en general, que siendo empleados y por su horario laboral o por la gran distancia para el desplazamiento desde su trabajo a la universidad, encuentran economía y flexibilidad dado que pueden interactuar con sus aulas de estudio, material interactivo, su docente tutor, los fines de semana o en las horas de la noche y con una buena disciplina pueden organizar mejor sus actividades tanto académicas, personales como las laborales.

De igual forma es un reto para estas entidades educativas porque el mercado ofrece diferentes alternativas tecnológicas, algunas están sólo probando algún sistema de gestión del aprendizaje a través de (Learning Management System -LMS) en el que pueden organizar y distribuir los materiales del curso, desarrollar foros de discusión, realizar tutorías, seguimiento y evaluación a los estudiantes; otras ya lo utilizan en su actividad diaria, otras han incorporado sistemas de gestión de contenidos (CMS), para soportar los LMS, y las últimas ya tienen sistemas de gestión de contenidos de aprendizaje (LCMS) y complementos como las bibliotecas digitales, sistemas de admisiones, registro y pagos en línea (Ortíz, 2007).

En este sentido, no se puede desligar el tema de convergencia tecnológica en cualquiera de estos ámbitos y más cuando el individuo por un contexto especial en el desarrollo de sus actividades cotidianas, exige un acceso a la información en cualquier momento, hora y lugar; por ello se debe contar con un dispositivo tecnológico diferente al de su oficina, de tal modo que le permita la conexión inmediata y efectiva para realizar las tareas deseadas ya sean laborales o académicas.

Por ello, la convergencia se está dando como respuesta a esta nueva realidad y a la necesidad de poder conectar 
todos los equipos para poder trabajar de forma cómoda y productiva tal y como se hace en puestos de trabajo. El estudio por ejemplo de Internacional Data Corporation (IDC) ${ }^{4}$, Predicciones IDC 2013 para Latinoamérica, mostró que una tercera parte de los trabajadores en Latinoamérica usan un teléfono móvil o Smartphone para fines de negocios, y dos de cada tres empresas movilizan a más del $10 \%$ de sus empleados con estos dispositivos o tabletas electrónicas. Cada vez más, las Notebooks, los SmartPhones y las tabletas son comprados por los consumidores y llevados a las empresas bajo BYOD ${ }^{5}$. El mismo estudio concluye también que en 2013 más de la mitad de los trabajadores usarán un dispositivo móvil con fines de negocios; y efectivamente es lo que está sucediendo en la actualidad; pese a esta situación, encuestas recientes de IDC muestran que no todas las empresas están debidamente preparadas para la velocidad en la que esta tendencia está evolucionando. Por tal motivo, es hora de comenzar a pensar en la convergencia de dispositivos de la mano de la movilidad como una inversión en pos de la productividad (Ruíz, sf).

Por lo anterior, tanto las empresas como las entidades educativas deben estar al tanto de los adelantos tecnológicos, lo cual exige el uso de dispositivos móviles así como el desarrollo de herramientas que se puedan trabajar desde éstos, facilitando las actividades cotidianas del ser humano que le permitan una mejor condición de vida profesional y laboral.

En este sentido, se debe resaltar que la tecnología constituye una parte importante, mientras los estudiantes con su perfil y disposición y, por supuesto, los docentes que también deben estar preparados para estos cambios, deben replantear los contenidos de sus asignaturas, su manera de llegar al estudiante en la didáctica, proporcionar las herramientas para el desarrollo de actividades, elaborar talleres o casos que el estudiante pueda desarrollar de forma apropiada y el docente efectivamente pueda evaluar el proceso de enseñanza-aprendizaje, provocando en el individuo su innovación, motivación, disciplina y compromiso para enriquecer sus conocimientos.

En los últimos tiempos, la sociedad ha experimentado una transformación acelerada entendida, en el pensamiento de muchos autores, como el nacimiento de una nueva sociedad cuyas expresiones más complejas se han sintetizado en lo que hoy se ha venido denominando de múltiples formas como sociedad digital, sociedad red, sociedad del conocimiento, sociedad de la información o era de la inteligencia (Ramírez, 2011).

Llegando a este punto en el contexto a estudiar, la Universidad Militar Nueva Granada en su continuo interés por capacitar a los miembros de las Fuerzas Militares, la Policía Nacional y sus familias, ha venido buscando alternativas de formación para el personal de oficiales, suboficiales y sus familias, que como consecuencia de los traslados a otras unidades y la situación de orden público, se han encontrado ante la imposibilidad de acceder a la educación superior y obtener un título profesional por el sistema presencial. (Salcedo, 2001).

En el primer semestre de 1996, la Universidad adquirió equipos con multimedia y organizó una sala de sistemas, conectándose al nodo de internet y a la red integrada de microondas del Ministerio de Defensa para permitir el desarrollo del programa de educación a distancia interactiva.

\section{Según el General Eduardo Herrera (2009):}

El fin primordial fue proyectar la UMNG entre las mejores instituciones de educación superior de Colombia y contribuir al desarrollo de los miembros del sector defensa además de ser "una Universidad con cobertura nacional e internacional, vinculada universalmente por redes virtuales a donde convergen toda clase de grupos de la sociedad ( $\mathrm{p}$. 22).

3. IDC. Empresa multinacional dedicada a la investigación de mercados en las áreas de tecnología de la información y telecomunicaciones.

4. BYODC, que significa raiga su propio dispositivo, por sus siglas en inglés Bring Your Own Device. 
La convergencia tecnológica en las aulas virtuales de la facultad de estudios a distancia de la universidad militar nueva granada (UMNG)

La Facultad de Estudios a Distancia (FAEDIS) se creó con la misión de contribuir a la capacitación profesional de todos los colombianos, priorizando a oficiales y suboficiales de las Fuerzas Militares y de la Policía Nacional; actualmente ofrece programas de pregrado en Administración de Empresas, Contaduría Pública, Relaciones Internacionales y Estudios Políticos, Ingeniería Civil e Ingeniería Industrial y a nivel de postgrado la Especialización en Alta Gerencia.

Actualmente uno de los propósitos tecnológicos, académicos y didácticos de la UMNG, para ofrecer cobertura, facilidad y disposición de la información para la formación de competencias en sus estudiantes, es reunir esfuerzos y dirigir recursos para invertir en temas de ubicuidad, conectividad y convergencia tecnológica.

En este proceso de actualización de los contenidos educativos para que puedan ser consultados y trabajados a través de los teléfonos inteligentes y tabletas electrónicas, se hace necesario por supuesto, conocer de la población objeto de estudio, características en los temas de conectividad, acceso a la información y actualización tecnológica móvil con las que median y realizan sus actividades académicas en el contexto B_Learning de la institución.

\section{Metodología de la investigación}

En esta investigación se aplicó una encuesta con un número de participantes de 3.291 usuarios (docentes, estudiantes y personal administrativo), se desarrollaron aquí 10 preguntas y se logró recolectar para análisis un total de 32.291 registros. El diseño metodológico se llevó a cabo mediante un estudio de caso, en donde se evaluaron las características de la población en su rol dentro del escenario B_Learning, el número de población por programa, la disponibilidad tecnológica con la que cuentan para el desarrollo de sus actividades académicas, enfocadas hacia la actualización tecnológica y la conectividad en el acceso móvil a sus contenidos de estudio.
El enfoque que enmarcó el estudio se hizo desde lo crítico social, pues la percepción de la población fue a través de este instrumento que permitió al individuo suministrar la información de acuerdo con su propio contexto tecnológico disponible y escenario B_Learning para el acceso a la información dentro de la plataforma Moodle.

Finalmente, la población se logró segmentar de la siguiente manera:

- Caracterizando a la población por rol, como está compuesto el escenario B_Learning.

- Caso 1. Categorizando a la comunidad de acuerdo con el tipo de conectividad con la que cuenta.

- Caso 2. Categorizando a la comunidad de acuerdo con los tipos de dispositivos móviles con los que tendría el acceso fácil a la información.

A continuación, se presenta el diseño metodológico para el desarrollo de esta investigación (véase figura 1).

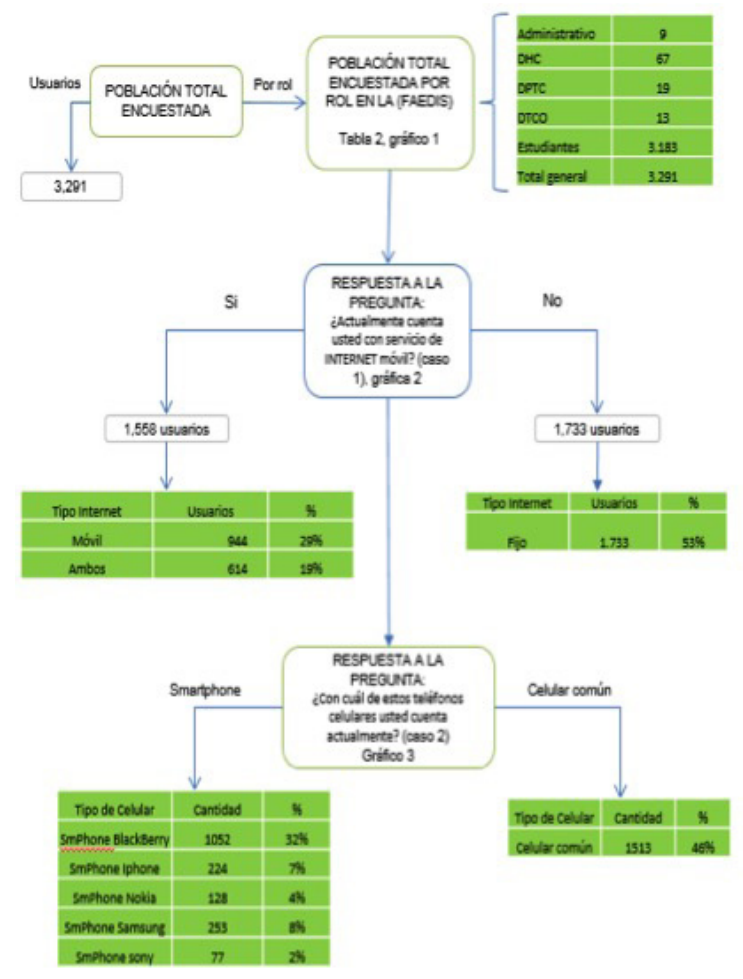

Figura 1. Diseño metodológico y alcance del artículo Fuente: El autor. 


\section{Resultados}

Los resultados permiten caracterizar la comunidad B Learning desde sus diferentes roles y desempeños en las actividades de enseñanza-aprendizaje; desde allí obtener la información relevante para determinar quiénes de ellos están preparados para la convergencia y disposición de contenidos a través de los dispositivos móviles, en este proceso académico a través de ambientes de aprendizaje mediado por las TIC.

\section{Características de la población B_Learning según su rol en la Facultad}

La población se caracteriza de acuerdo con el rol en la Facultad. Se identifica el personal administrativo que ofrece apoyo para que los procesos académicos tengan continuidad; se observan los conceptos y percepciones de los docentes de hora cátedra (DHC), docentes de planta tiempo completo (DPTC) u ocasionales (DTCO) y los estudiantes de todos los programas y semestres (véase tabla 2).

Tal como se muestra en la figura 2, los usuarios con rol de funcionarios administrativos, DHC, DPTC y DTCO tuvieron representación baja frente al total de estudiantes; sin embargo, la población encuestada con estos roles fue equivalente a un censo, pues fue el total activo en el LMS. Por otro lado, la representación de estudiantes da una equivalencia del $96,72 \%$ de la población a quien en especial va dirigido el estudio,

El diseño metodológico corresponde a las siguientes preguntas:

Caso 1. Categorizando a la comunidad de acuerdo con el tipo de conectividad con la que cuenta.

Pregunta: ¿Actualmente cuenta con servicio de internet móvil?

Analizando los resultados en el figura 3, se puede apreciar que un $29 \%$ de la población cuenta con servicio de internet móvil, un $19 \%$ disponen de conectividad conjunta fija y

\begin{tabular}{|c|c|c|}
\hline Rol & Usuarios & $\%$ \\
\hline Administrativo & 9 & $0,27 \%$ \\
\hline DHC & 67 & $2,04 \%$ \\
\hline DPTC & 19 & $0,58 \%$ \\
\hline DTCO & 13 & $0,40 \%$ \\
\hline Estudiante & 3183 & $96,72 \%$ \\
\hline Total general & 3291 & $100,00 \%$ \\
\hline
\end{tabular}

Tabla 2. Cantidad de usuarios encuestados por rol Fuente: El autor.

\section{Población encuestada 3.291 usuarios Moodle}

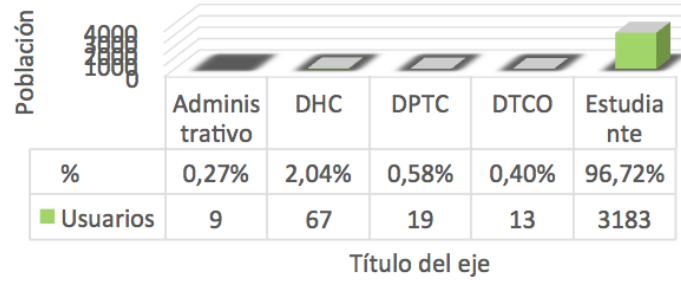

Usuarios \%

Figura 2. Cantidad de usuarios encuestados por rol Fuente: El autor.

Conectividad a Internet de la población académica

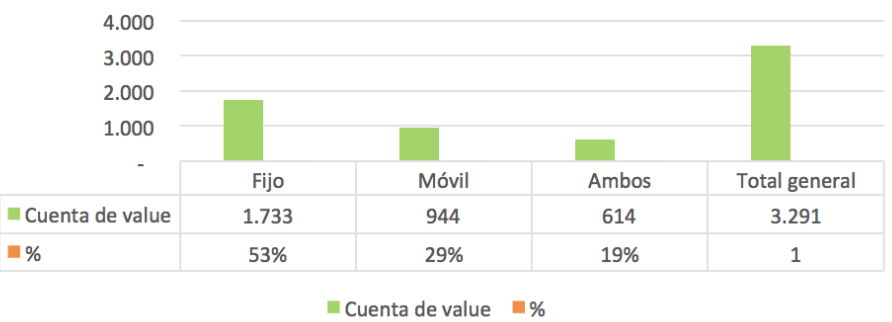

Figura 3. Tipos de conectividad disponibles en la población Fuente: El autor.

móvil; mientras que un gran porcentaje de la población equivalente a $53 \%$ sólo tiene conectividad a internet fija, significa que el acceso a contenidos mediante dispositivos móviles es limitado, prácticamente para la mitad de la población. 
La convergencia tecnológica en las aulas virtuales de la facultad de estudios a distancia de la universidad militar nueva granada (UMNG)

Caso 2. Categorizando a la comunidad de acuerdo con el tipo de teléfono móvil con los que tendría el acceso fácil a la información.

Pregunta: ¿Con cuál de estos teléfonos celulares cuenta actualmente?

Tipos de dispositivo celular de la comunidad académica

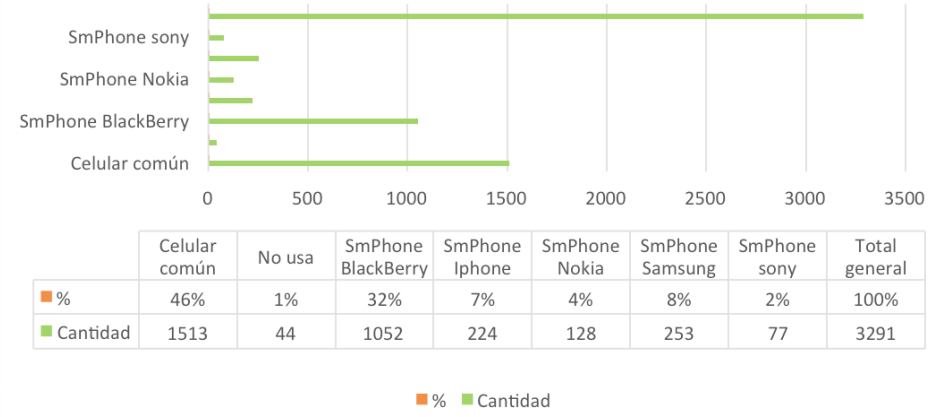

Figura 4. Tipos de dispositivos móviles disponibles en la población Fuente: $\mathrm{El}$ autor.

Analizando los resultados en la figura 4, se puede apreciar que la población en general equivalente a un total de 53\%, dispone de un teléfono SmartPhone que permitiría el acceso en hardware a contenidos diseñados y desarrollados para tal fin; por otro lado, el $46 \%$ de la población tienen teléfonos comunes, lo que se debería en este aspecto proceder a una actualización tecnológica para apoyar prospectivamente el acceso de esta población a contenidos de última generación.

\section{Conclusiones}

El diseño metodológico planteado permitió el levantamiento de información de una población de 3.291 usuarios activos en la plataforma Moodle, quienes desarrollan procesos de apoyo, docencia y aprendizaje virtual en la Facultad de Estudios a Distancia (FAEDIS) de la Universidad Militar Nueva Granada; permitiendo la categorización de la comunidad por rol dentro de la Facultad, resaltando el predominio de estudiantes (3.183) con el $96.72 \%$ del total de la comunidad encuestada.

Respecto al acceso de información por internet móvil, la mayoría de la comunidad académica no está preparada para recibir información en cualquier lugar donde la persona se encuentre, esto equivale a que el 53\% de usuarios tienen solamente acceso a contenidos desde la plataforma Moodle, de una ubicación fija (en un puesto de trabajo); por tanto, es importante diseñar estrategias que permitan el acceso para continuar con la migración hacia este propósito por parte de la institución y que permita beneficiar al 100\% de su comunidad académica.

En hardware de dispositivos celulares adaptados para estas tecnologías, se encontró que el 53\% de la población, dispone de un teléfono inteligente, que le permitiría el acceso a contenidos diseñados para estos artefactos. No obstante, también el 46\% (1.512) de los usuarios tendrían que actualizar tecnológicamente sus equipos para acceder a información de contenidos diseñados para este fin. Es decir, esta población, no tendría acceso desde sus dispositivos móviles a los contenidos diseñados para soportar la ubicuidad y convergencia tecnológica.

La introducción de los entornos virtuales en los dispositivos móviles promueve un nuevo sistema de estudio, pero debe ser un proceso progresivo, que ocasiona cambios en la metodología de estudio, los recursos pedagógicos, y las prácticas educativas; ya sea porque genera cierto tipo de resistencia, dificultades técnicas, siendo un proceso formativo diferente y que exige disciplina, dedicación y actualización tecnológica por parte de los usuarios.

\section{Referencias}

Alarcón, M. y Lorenzo, C. (mayo-agosto de 2012). "Diferencias entre usuarios y no usuarios de redes sociales virtuales en la Web 2.0". En: Revista Venezolana de Información, Tecnología y Conocimiento, 9(2), 31-49.

Burkle, M. (2011). “El aprendizaje on-line: oportunidades y retos en instituciones politécnicas". En: Revista Científica de Educomunicación, XIX(37), 45-53.

García, A. (2001). La educación a distancia. Barcelona: Ariel. 
Gómez, P. y García, M. (2011). "Nativos digitales y nuevas tecnologias: implantación en la Universidad”. En: Educação Temática Digital, 12, 73. Consultado en: http://ezproxy.umng.edu.co:2048/ login? url=http://search.proquest.com/docview/1 442219766 ? accountid $=30799$.

Herrera, E. (2009). La UMNG que imaginamos: Diez escenarios para sustanciar. Bogotá.

Ortega, I. (2004). "Los cursos virtuales en la Universidad Nacional de Educación a Distancia: posibilidades didácticas". En: Enseñanza, 391 - 412.

Ortíz, L. (junio de 2007). "Campus Virtual: la educación más allá del LMS". En: Revista e Universidad y sociedad del Conocimiento, 4(1), 1-7.

Ramírez, M. (2009). "Recursos tecnológicos para el aprendizaje móvil (mLearning) y su relación con los ambientes de educación a distancia: implementaciones e investigaciones". En: RIED, 2(1), $57-82$.
Ramírez, Y. (2011). "Presentación de contenidos en entornos educativos virtuales basada en la adaptación a los estilos de aprendizaje". En: Revista Eticanet, 306-321.

Ruíz, C. (s.f.). Ministerio de las Tecnologías de la Información y las Comunicaciones. Recuperado el 25 de Febrero de 2014 de la página web MINTIC: http://www.mintic.gov.co/portal/604/w3-article-5111.html

Salcedo, A. (2001). Reseña histórica Facultad de Educación a Distancia. Bogotá, D.C: Guadalupe Ltda.

Santoveña, S. (2006). "Análisis de cursos de educación social, los entornos virtuales de aprendizaje y su incidencia en la calidad". En: Universidad Nacional de Educación a Distancia (España).

Vargas, I. (2010). "Beneficios del modelo de la Universidad Virtual". En: Noticias Financieras. 